\title{
La Lista de nombres vulgares y botánicos de árboles y arbustos propicios para repoblar los bosques de la República de Fernando Altamirano y José Ramírez a más de 110 años de su publicación
}

\section{The List of common and botanical names of trees and shrubs suitable for restoration of the woods of the Republic by Fernando Altamirano and José Ramírez, more than 110 years after its publication}

\author{
Ma. Hilda Flores Olvera ${ }^{1 *}$ y Roberto Lindig-Cisneros ${ }^{2}$ \\ ${ }^{1}$ Instituto de Biología, Universidad Nacional Autónoma de México. Apartado postal 70-233. C.P. 04510 \\ México, D.F. \\ *Correspondencia: mahilda@servidor.unam.mx \\ ${ }^{2}$ Laboratorio de Ecología de Restauración, Facultad de Biología, Universidad Michoacana de San \\ Nicolás de Hidalgo, Apartado postal 18, Admin. 3, Santa María, 58091 Morelia, Michoacán, México.
}

\begin{abstract}
Resumen. Dado el valor histórico y práctico de la lista de árboles y arbustos para repoblar los bosques de la República Mexicana que Altamirano y Ramírez publicaron hace 111 años y de la inaccesibilidad para consultarla, se elaboró una lista con los nombres científicos corregidos y/o los sinónimos actualizados, en su caso, para facilitar la consulta. Se proponen las posibles especies a las que Altamirano y Ramírez se refirieron, y para las que sólo indicaron nombre vulgar y género. Se anota si las especies son nativas o introducidas, su uso en el presente y los métodos de propagación. Se analiza y discute la lista a la luz de los enfoques actuales de restauración. El documento original consta de 281 nombres vulgares que representan 259 especies de 72 familias; 231 especies son angiospermas y 28 gimnospermas. La mayoría de los géneros tienen una sola especie y tan solo nueve, más de cuatro: Pinus con 19, Quercus con 11, Cordia y Tabebuia con cinco, Annona, Bursera, Caesalpinia, Juniperus y Yucca con cuatro. Se reproduce la carátula y la última página de la edición de 1894.

Palabras clave: árboles, arbustos, restauración, México.

Abstract. Because of the importance of the list of plant species for repopulating the forests of the Mexican Republic, first published by Altamirano and Ramírez 111 years ago, and the difficulty to access it, a list is presented considering current trends in ecological restoration, and corrected scientific names were added to facilitate its use. To improve the utility of the list some information such like if the species are native or introduced, their uses and propagation methods is provided. The authors provide 281 common names that represent 259 species of 72 families of which 231 species are angiosperms and 28 gymnosperms. Most of the genera are represented by one species and only nine genera are represented by four or more species: Pinus (19 species), Quercus (11 species), Cordia and Tabebuia (5 species each) and Annona, Bursera, Caesalpinia, Juniperus and Yucca (4 species each). The front and last pages are presented as originally published.
\end{abstract}

Key words: trees, shrubs, restoration, México.

\section{Introducción}

A finales del siglo XIX, las investigaciones que se desarrollaron en el Instituto Médico Nacional dieron un fuerte impulso a la actividad botánica en México. Entre los personajes dedicados al estudio de las plantas, Fernando Altamirano y José Ramírez destacaron por sus contribuciones en diversos campos de la botánica, como etnobotánica, florística, taxonomía, etc., sin dejar de lado contribuciones prácticas vinculadas con las necesidades del país (Flores Olvera y Ochoterena-Booth, 1991). Tal es el caso de "La lista de nombres vulgares y botánicos de árboles y arbustos propios para repoblar los bosques de la
República" que Altamirano y Ramírez publicaron en 1894 (figura 1).

Esta contribución se repartió en forma de folleto durante el segundo Congreso Médico Mexicano en noviembre de 1894 (Flores Olvera y Ochoterena-Booth, 1991); consiste de 281 nombres vulgares de plantas con los respectivos nombres científicos, la manera de reproducción y el clima en donde crecen. No lleva una presentación ni la bibliografía utilizada por los autores. En Sinonimia vulgar y científica de las plantas mexicanas (Ramírez y Alcocer, 1902), José Ramírez explicó que la lista derivó de la que G. Crespo y él mismo publicaron en Paris en 1889, con los nombres vulgares y científicos de los árboles identificados 
hasta esa fecha y de los cuales se habían enviado colecciones de maderas a la Exposición Universal, y a la que se habían agregado las identificaciones realizadas en los siguientes cinco años. En esa forma apareció en Progreso de México, vol. 11 de 1904, pp. 235, 336, 524 y 747 y vol. 12 de 1905, pp. 197. En la biblioteca de la Universidad de Chapingo se conservan algunos números de esta revista, pero desafortunadamente ninguno de estos dos. Posteriormente, Maximino Martínez (1927) la retomará para actualizarla y enriquecerla, publicándola como el conocido "Catálogo de nombres vulgares y científicos de plantas mexicanas".

Durante muchos años, la lista de Altamirano y Ramírez (1894) ha permanecido ignorada por la comunidad científica a pesar de su gran relevancia, hoy tal vez más que entonces, debido al nivel de deterioro que presentan muchos de los ecosistemas del país. Probablemente éste fue el primer esfuerzo por crear una lista de las especies de plantas útiles para reforestación y, diríamos hoy en día, restauración ecológica. Después de Altamirano y Ramírez, otros autores se preocuparon por el potencial de las especies nativas para ser utilizadas en proyectos de esta naturaleza. Cabe destacar la contribución de Conzatti (1914), sobre especies principalmente nativas útiles para reforestar el Valle de Oaxaca y los esfuerzos más recientes de Vázquez-Yanes et al. (1999) y de Benítez et al. (2004).

\section{Método}

Después de una búsqueda en diversas bibliotecas de la ciudad de México y del extranjero, sin éxito, la lista se localizó en microfilm a través del sistema de bibliotecas de la Universidad de Wisconsin-Madison (SOLINET/ASERL Cooperative Microfilm Project, NEH PS-20317) de la cual se solicitó una copia impresa, que en general se encuentra en buen estado con excepción de la página cuatro, donde parte del texto es ilegible, posiblemente porque el original del cual se obtuvo el microfilm se encontraba ya dañado.

Se presenta una nueva lista, basada en la de Altamirano y Ramírez (Apéndice). Para facilitar la consulta, se agregó una columna con las correcciones de los nombres científicos y/o los sinónimos actualizados; debajo de los nombres de las familias como fueron anotados por los autores, el nombre corregido; se indica si las especies son nativas de México o introducidas, los diferentes tipos de propagación, los usos registrados en la literatura consultada, y si están consideradas en las obras recientes de Vázquez-Yanes et al. (1999) y Benítez et al. (2004).

Para la corrección de la nomenclatura y/o actualización de la sinonimia se consultaron diversas floras regionales como Standley y Steyermark (1946), Shreve y Wiggins (1964), Correll y Johnston (1979), Hickman (1993), Rzedowski y Calderón de Rzedowski (1991-2003), Stevens et al. (2001), Flora de Veracruz (1978-2003), Flora Neotropica (1967-1999), McVaugh (1983-1993), Rzedowski, Rzedowski y colaboradores (2001). También se consultaron Standley (1920-1926), McVaugh y Rzedowski (1964-1966), Hortus Third (1976), Zanoni y
Adams (1979), Gleason y Cronquist (1991), así como International Plant Name Index Query en la dirección http:/www.ipni.org/ipni/query_ipni.html y W3Tropicos en http://mobot.mobot.org/W3T/Search/vast.html. Para sugerir las posibles especies listadas por Altamirano y Ramírez por nombre común y género se consultaron Ramírez y Alcocer (1902), Standley y Steyermark (1946), Martínez (1927), Pennington y Sarukhán (1998) y Benítez et al. (2004). Por su valor histórico, se reproduce la carátula y la última de las páginas de la lista de Altamirano y Ramírez tal como fue editada en 1894 (figuras 1, 2).

\section{Resultados}

Análisis de la lista. La lista original consta de 281 nombres vulgares pero ya que una de las páginas se encuentra muy dañada en la versión recuperada, nueve nombres vulgares son ilegibles, pudiéndose leer los nombres científicos de cuatro de ellas (figura 3). De acuerdo con los nombres científicos originalmente provistos por Altamirano y Ramírez (1894) se incluyen 58 familias y 161 géneros de los cuales 48 cuentan con más de una especie enlistada. Después de actualizar la nomenclatura, la lista incluye 259 especies de 72 familias, de las cuales 231 son angiospermas y 28 gimnospermas. La mayoría de los géneros tienen una sola especie y tan solo nueve tienen más de cuatro especies, destacando Pinus con 19, Quercus con 11, Cordia y Tabebuia con cinco, Annona, Bursera, Caesalpinia, Juniperus y Yucca con cuatro.

La lista original tiene diversos errores en los nombres científicos, muchos de ellos evidentemente de mecanografía y ortográficos. También hay errores de citación de las autoridades de los nombres. Por ejemplo, el "sumaque" se enlista como Rhus mollis L. (Linnaeus) pero esta autoridad del nombre no es conocida en la literatura taxonómica a pesar de que se encontraron cuatro homónimos. Rzedowski, Rzedowski y colaboradores (2001) citan R. standleyi F.A. Barkley (=R. mollis Kunth) como "sumaco". En la lista, Cereus giganteus se adjudica a Zuccarini (Zucc.), mientras que la autoridad de la especie es Engelmann (Engelm.) y Swietenia mahagoni a Linn. (Linnaeus), mientras que la autoridad es Lamarck y sinónimo de S. mahagoni (L.) Jacq. Salacia elliptica fue atribuida a Peir (Peyritsch), mientras que la autoridad es (Mart.) G. Don; es interesante que Peyritsch describió tres variedades de la especie, por lo que es posible que Altamirano y Ramírez se refirieran a alguna de ellas.

Además, es probable que en los nombres vulgares también haya errores. Así, por ejemplo en la lista, la "flor del papagallo" es una especie dudosa de Piscidia, mientras que Ramírez y Alcocer (1902) llaman "flor de papagallo" a $P$. erytrina L. (=P. piscipula (L.) Sarg.). Así mismo, el "copalxocotl" se señala como especie dudosa de Sapindus, mientras que Ramírez y Alcocer (1902) enlistan al "copalxocote" con la misma identidad taxonómica; el "xochicopal" se refiere a una especie dudosa de Bursera, mientras que Ramírez y Alcocer (1902) listan como 

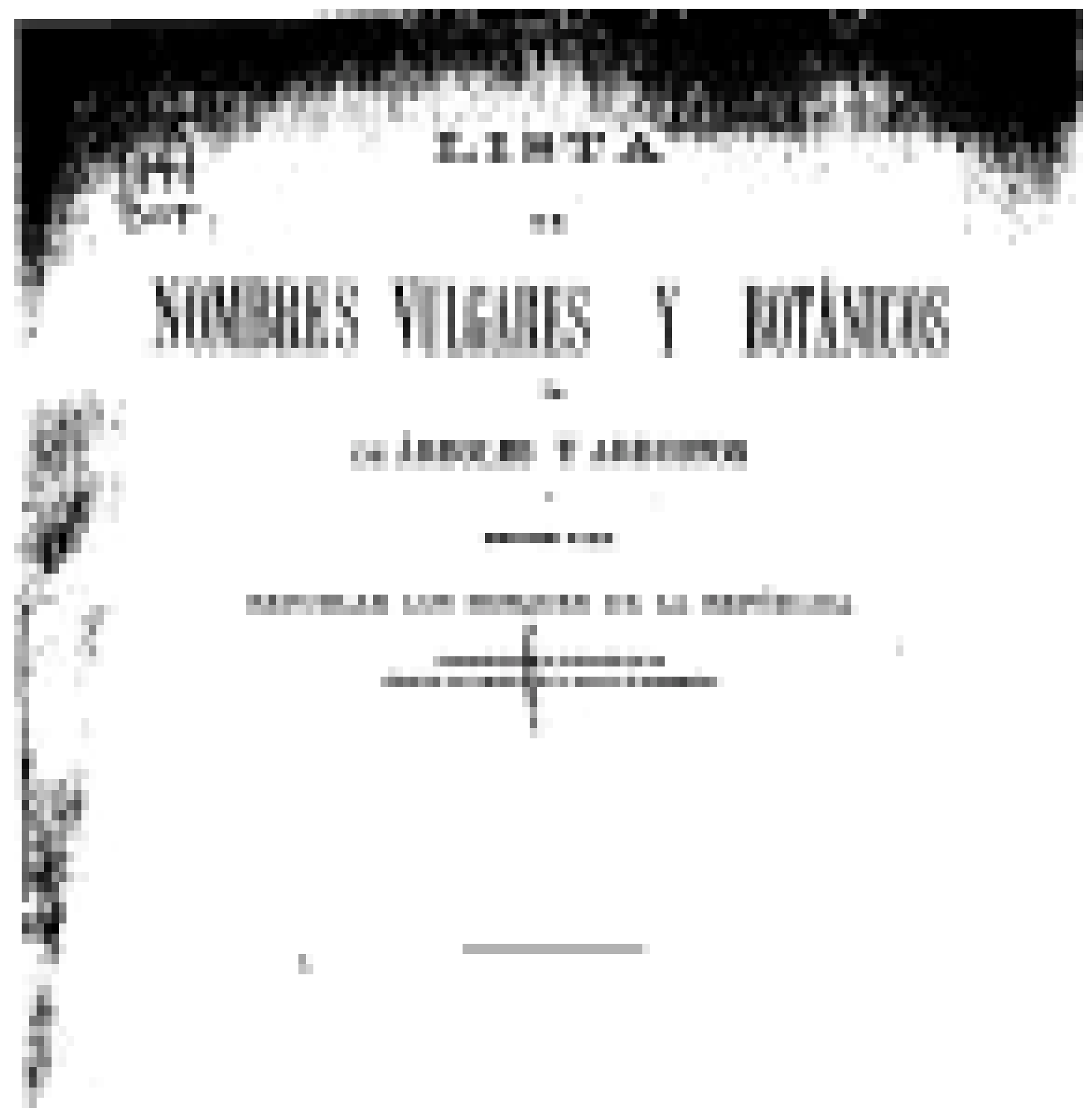

1.

\section{배마패}

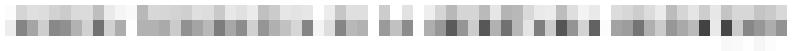

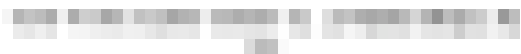

IIII H

Figura 1. Carátula de La lista de nombres vulgares y botánicos de árboles y arbustos propios para repoblar los bosques de la República de Altamirano y Ramírez (1894). 
Flores y Lindig- Cisneros.- Lista revisada de árboles y arbustos de Altamirano y Ramírez

"xochicopalli" a B. mexicana Engl. (=Elaphrium mexicanum (Engl.) Rose). En la lista el "nananchi o nancinxocotl" es una especie dudosa de Malpighia, mientras que Ramírez y Alcocer (1902) listan "nananche" y "nanzinxócotl" para Byrsonima cotinifolia Kunth (=B. crassifolia (L.) Kunth) pero no para alguna especie de Malpighia; ambos géneros son de la familia Malpighiaceae.

Este último aspecto denota, además, que Altamirano y Ramírez (1894) al momento de publicar la lista tenían dudas en la identificación del género e incluso de la familia, de algunas plantas como sería de esperarse por la escasa literatura taxonómica disponible en ese momento para plantas mexicanas. Así, por ejemplo, Standley (1920) aclaró que Ficus petiolaris H.B.K. había sido registrada para México frecuentemente como $F$. nymphaeifolia L., especie Sudamericana, por lo que podríamos considerar una corrección en la identificación ya que, además, entre los nombres vulgares de $F$. petiolaris incluye "higuerón" y "tescalamate", que dieron también Altamirano y Ramírez. Sin embargo, ya que Standley también indica que $F$. cotinifolia H.B.K. recibe en México el nombre de " higuerón", no hay seguridad a cual especie se refirieron.

En la "Sinonimia vulgar y científica de las plantas mexicanas" (Ramírez y Alcocer, 1902) encontramos evidencia de los avances en la identificación de algunas plantas que implicaron correcciones de nombres científicos publicados por Altamirano y Ramírez ( 1894). Así, por ejemplo, mientras que en esta última el "guanábano" fue sugerido como una especie de Annona, en 1902 fue identificada como Annona muricata L.; el "madroño borracho" fue sugerida en la lista original como una especie de Arbutus, mientras que en 1902 fue identificada como Arctostaphylos arguta (Zucc.) DC. (=Comarostaphylis discolor (Hook.) Diggs); ambos géneros de la familia Ericaceae. El "cuajiote colorado" sugerido en la lista como una especie dudosa de Rhus (Anacardiaceae) fue enlistado en 1902 como Bursera fagaroides Engl. (Burseraceae). En la lista corregida sugerimos los nombres científicos de Ramírez y Alcocer (1902) que corresponden con el único nombre vulgar incluido en su trabajo y el indicado por Altamirano y Ramírez. Dada la inseguridad en la identificación de Ramírez y Alcocer (1902) del "teponaxtle o teponaxtli" como una especie de Bursera (Burseraceae) y aunque coincide con el "teponaxtle" de Altamirano y Ramírez que fue identificado como una especie dudosa de Amyris (Rutaceae), no se incluye en la lista corregida que presentamos. Ramírez y Alcocer enlistaron varias especies distintas para algunos nombres vulgares que fueron usados por también por Altamirano y Ramírez para las cuales sugirieron una posible identificación genérica. Por ejemplo, Ramírez y Alcocer (1902) enlistaron el "rosadillo" tanto como Swietenia mahogani L. como para una especie dudosa de Cedrela, ambas de la familia Meliaceae, como fue considerada por Altamirano y Ramírez. Así mismo, Ramírez y Alcocer (1902) enlistan "quiebra-hacha" tanto bajo
Hymenea (Leguminosae) como bajo especie dudosa de Guayacum (Zygophyllaceae) al igual que Altamirano y Ramírez. Ya que es imprecisa la aplicación de estos nombres científicos sin mayor información no los incluimos en la lista corregida. Para otras plantas como "cueramo", "huacalillo","laurelillo agrio", "macuile", "salate", etc. Ramírez y Alcocer (1902) no habían logrado avance en la identificación a especie.

Otros autores han consignado los mismos nombres vulgares de Altamirano y Ramírez (1894) pero dada la conocida aplicación del mismo nombre vulgar a plantas inclusive de familias distintas, tampoco los incluimos en la lista corregida. Por ejemplo, Martínez (1927) enlista "tetlatia" como Comocladia engleriana Loes, mientras que Altamirano y Ramírez la incluyen como especie dudosa de Rhus, ambas Anacardiaceae. Del mismo modo, Martínez (1927) y Pennington y Sarukhán (1998) refieren el "cocuite"como Gliricidia sepium (Jacq.) Steud. y Piscidia piscipula (L.) Sarg., mientras que Altamirano y Ramírez la consideran especie dudosa de Robinia; los tres géneros corresponden a la familia Leguminosae.

En algunos casos Altamirano y Ramírez (1894) proponen dos veces la misma especie. Por ejemplo, listaron Ulmus mexicana bajo los nombres vulgares de olmo o cempoalehuatl (consignados por Benítez et al., 2004), mientras que tenían duda de si el nombre "papalote" correspondía a la misma especie. Este último nombre vulgar fue consignado por Pennington y Sarukhán (1998) para la misma especie, lo cual podría esclarecer la identidad de la especie a que se referían Altamirano y Ramírez (1894).

En otros casos, al hacer la búsqueda bibliográfica de especies que son conocidas con los nombres vulgares que concordaran con el género para el que Altamirano y Ramírez (1894) desconocieron la especie, se propone una especie ya listada. Este es el caso de Cordia alliodora que Pennington y Sarukhán (1998) consignan como "cueramo", mientras que Altamirano y Ramírez la consideraron como especie desconocida de Cordia. Además, la especie conocida como "tambor" fue identificada por Altamirano y Ramírez como Cordia gerasscanthus Jacq. que es un sinónimo de Cordia alliodora. También es el caso para Bursera aloexylon citada por Altamirano y Ramírez (1894) como "linaloe" y como especie desconocida de Bursera bajo el nombre "xochicopal, citado por Martínez (1927) para la misma especie. Por su parte, Ficus pertusa resulta citada dos veces ya que $F$. complicata dado por Altamirano y Ramírez para "amesquite y samatito" y F. padifolia dado por Ramírez y Alcocer (1902) para el "camichin" son sinónimos del primero.

Es importante destacar que al actualizar los nombres científicos de la lista de Altamirano y Ramírez (1894), algunos resultan en sinónimos de especies citadas con los mismos u otros nombres vulgares. En el primer caso esta Talauma macrocarpa que es un sinónimo de $T$. mexicana; ambas fueron listadas como "yoloxóchtl". En el segundo caso esta Cedrela dugesii que fue referida como 


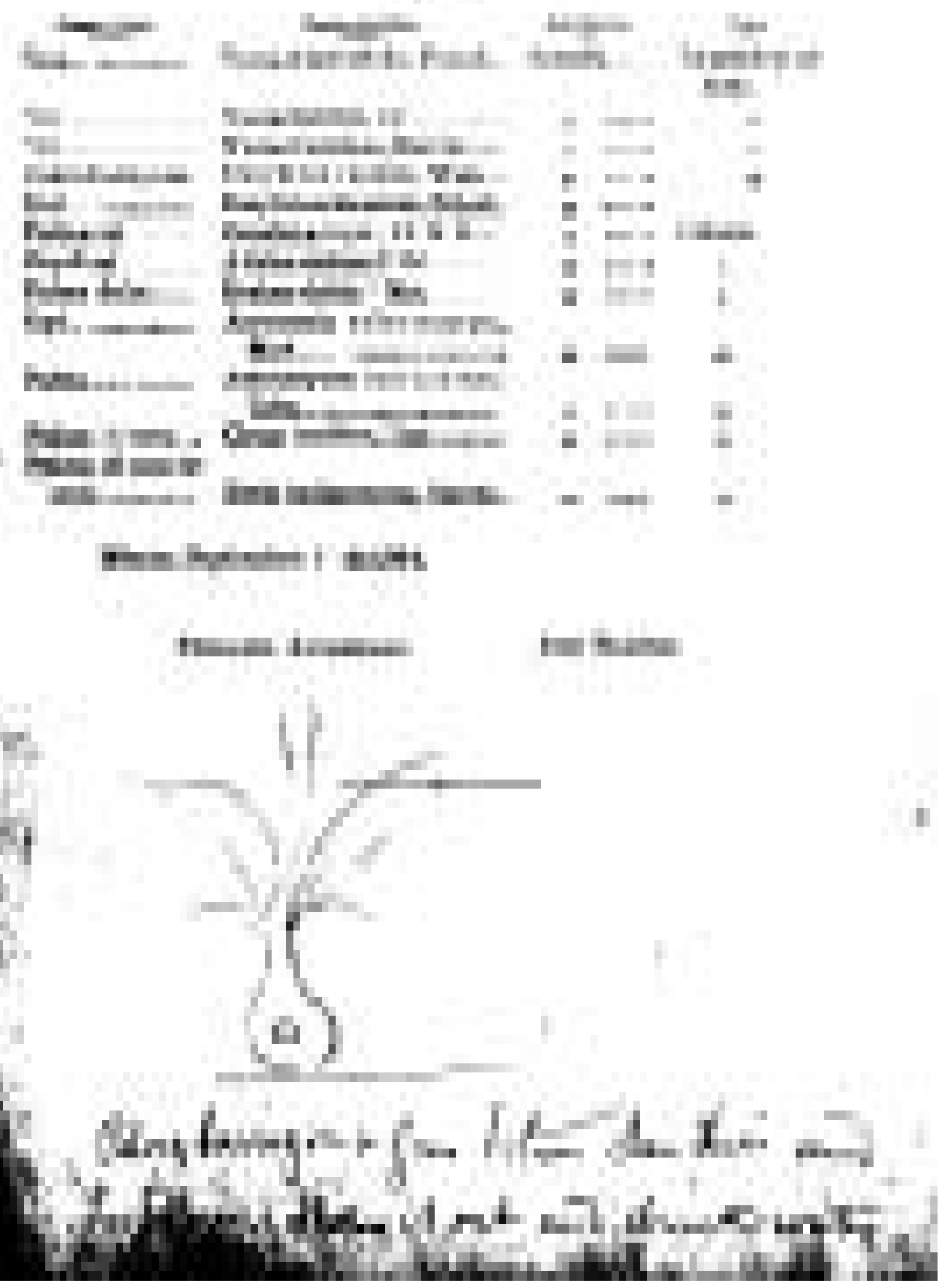

Figura 2. Última página de La lista de nombres vulgares y botánicos de árboles y arbustos propios para repoblar los bosques de la República de Altamirano y Ramírez (1894). 
"cedro" y que es un sinónimo de $C$. odorata, que a su vez fue consignada como "cedro macho". Tabebuia pentaphylla Hemsley citada como "roble de San Luis Potosí" es un sinónimo de $T$. rosea (Bertol.) DC. consignada también como "palo de rosa" por Altamirano y Ramírez (1894). A su vez, Persea gratisima Gaertn. (aguacate común) y $P$. drymifolia Schltdl. \& Cham. (aguacate oloroso) son sinónimos de $P$. americana Mill., mientras que Cupressus benthami Endl. (ciprés) y C. lindleyi Klotzsch ex Endl. (ciprés o cedro blanco) son sinónimos de C. lusitanica Mill.

Para algunas plantas de las que Altamirano y Ramírez (1894) indicaron el género, no es posible proponer la especie pues en la actualidad diversas especies del género se conocen con el mismo nombre vulgar. Tal es el caso por ejemplo del "amate", nombre con el que se conocen Ficus cotinifolia Kunth, $F$. petiolaris Kunth y F. tecolutensis (Liebm.) Moq.

La lista de Altamirano y Ramírez (1894) también incluye información sobre la técnica de propagación de las especies en función de tres categorías. La mayoría, o sea 262 especies, se propagan por semilla, mientras que once especies se propagan por medios vegetativos; diez por estaca y semilla (Cereus giganteus, C. stramineus, Erythrina corallodendron, Opuntia tuna, Populus balsamifera, $P$. canadensis, $P$. mexicana, Salix bonplandiana, $S$. humboldtiana y $S$. nigra) y una especie por retoño (Yucca aloifolia). Destacan las especies de los géneros Salix y Populus entre las que se propone su reproducción por estaca. Hoy en día se han desarrollado métodos de propagación vegetativa para muchas de las especies que Altamirano y Ramírez (1894) consideraban que se propagaban sólo por semilla (ver en la lista con nombres corregidos la columna de "Experiencia en restauración" en donde se incluye el tipo de propagación reportado con las siguientes claves $\mathrm{P}=$ propagación y entre paréntesis $\mathrm{s}=$ semilla, $\mathrm{v}=$ vegetativa).

En términos de los tipos de clima en donde crecen las especies enlistadas, los autores reconocen 7 tipos: caliente (152 especies), caliente y templado (15 especies), frío (46 especies), frío y templado (13 especies), templado (16 especies), templado y caliente (20 especies) y, templado y frío (17 especies). De estos siete tipos es imposible saber cual es el criterio de clasificación, en particular si los tipos "frío y templado" y "templado y frío" son equivalentes; la misma duda surge con los tipos "caliente y templado" y "templado y caliente". Cuando se consideran a las especies que se enlistan para las categorías "caliente y templado" y "templado y caliente" y se comparan con las especies incluidas en estas categorías mencionadas por Rzedowski (1986), se puede apreciar que corresponden a las categorías de bosque tropical perennifolio, bosque tropical caducifolio, bosque espinoso e incluso bosque mesófilo de montaña. Por ejemplo, Cordia dodecandra que se encuadra en la categoría de "caliente y templado" ha sido referida para una zona cubierta de bosques secundarios que originalmente correspondían a bosque tropical caducifolio (Rzedowski,
1978), mientras que Morus celtidifolia, que corresponde a la categoría de "templado y caliente" es reportado para el bosque mesófilo de montaña por este autor. Lo anterior impide llegar a una equivalencia entre los tipos de clima utilizados por Altamirano y Ramírez y otras clasificaciones, incluso si se consideran las otras categorías.

La mayor parte de las especies consideradas por Altamirano y Ramírez son nativas de México, incluyendo endémicas como Magnolia dealbata o M. schiedeana. Llama la atención que los autores hayan considerado especies silvestres no nativas de México como Protium heptaphyllum, Stigmaphyllon dichotomum, Stemmadenia gradiflora, Tabebuia obtusifolia de Centro o/y Sudamérica e incluso Casearia guianensis y Hura crepitans que también se distribuyen en las Antillas. Además, se incluyeron especies de distribución en el sur de los Estados Unidos de Norteamérica como Fraxinus cuspidata, F. greggii y Yucca baccata. Se enlistan 43 especies introducidas entre las que destacan: Anacardium occidentale, Annona cherimola, Carica papaya, Cinchona calisaya, Citrus aurantium, Citrus limonia, Citrus limetta, Cocos nucifera, Eucaliptus globulus, Hevea guyanensis, Mangifera indica, Melia azedarach, Mespilus germanica, Opuntia tuna, Schinus molle, Swietenia mahogany, Tamarindus indica y un cultivar Populus canadensis obtenido por hibridación.

Dada la inexistencia de una presentación de la lista que nos ocupa no esta claro si Altamirano y Ramírez (1894) pretendían introducir especies tales como Opuntia tuna, que es una especie de Las Antillas, habiendo en México especies nativas del género. En el mismo caso esta la caoba Swietenia mahagoni propuesta en la lista pero no mencionan a $S$. macrophylla King que es la especie nativa. ¿Altamirano y Ramírez verdaderamente pretendían introducirlas o simplemente pusieron ese nombre creyendo que se trataba de especies nativas? La lista no proporciona información al respecto y es posible plantear varias hipótesis aunque no sea posible discriminar entre ellas. En primer lugar, es posible que los autores ignoraran el origen de algunas de las especies considerando las limitaciones de acceso a información de la época, o que cuando la lista fue elaborada no había una preocupación por la posible introducción de especies invasoras, y por lo tanto asegurarse del origen geográfico de las especies no era una prioridad. Otra posibilidad es que los autores hayan considerado especies que a finales del siglo XIX eran de importancia económica, por ejemplo Hevea guyanensis, o que dieran alternativas de uso para los sitios reforestados. Esto se apoya en el hecho de que muchas de las plantas consideradas en la lista tienen algún valor como especies comestibles, medicinales como Cinchona calisaya de la que se crearon plantaciones por su efecto contra la malaria, de ornato e inclusive algunas de ellas con conocido uso prehispánico como para la elaboración de papel (amates) o fibras (pinos), así como hule y colorantes. También se deben considerar problemas de identificación ya mencionados, como sería el caso de Ficus nymphaeifolia L., o bien el caso de Ostrya virginica 
Willd. (=O. virginiana (Mill.) K. Koch, especie de los Estados Unidos de Norteamérica, mencionada por Altamirano y Ramírez como guapaque, mientras que Standley reportó para México como guapaque a Ostrya guatemalensis (Wink1.) Rose, distribuida en el sur de México, Guatemala y Costa Rica.

La inspección de la lista de Altamirano y Ramírez (1894) permite reconocer 26 leguminosas, especies de gran importancia para la recuperación de la fertilidad del suelo y que en muchos casos tienen la capacidad de establecerse en sitios con altos niveles de degradación (Bradshaw, 1982; Stopes et al., 1996; Ibewiro et al, 2000). De hecho, la familia Fabaceae y las coníferas (28 especies) son las que se encuentran representadas por un mayor número de especies en la lista.

A diferencia de la lista de Altamirano y Ramírez (1894), las obras de Vázquez-Yanes et al. (1999) y Benítez et al. (2004), esfuerzos de gran relevancia realizados a más de un siglo de distancia, incluyen información detallada sobre los métodos de propagación para la mayoría de las especies que enlistan, resultado del esfuerzo en este campo desde la publicación de la lista de Altamirano y Ramírez. Los trabajos recientes incluyen también los usos y el hábitat de las especies consideradas. En términos cuantitativos, es importante hacer notar que estas tres obras difieren en el número de especies consideradas y el tratamiento de las mismas, pues Vázquez-Yanes et al. (1999) incluyen fichas detalladas para 70 especies mientras que Benitez et al. (2004) lo hicieron para 107. En contraste, Altamirano y Ramírez enlistan 281 plantas pero la información que proporcionan es limitada. En estas tres obras hay una gran coincidencia en cuanto a muchas de las especies propuestas. VázquezYanes et al. (1999) comparten con Altamirano y Ramírez 28 especies, considerando las correcciones a la lista original realizadas en el presente trabajo, mientras que Benítez et al. comparten 61 (ver columna de comparación entre listados en la lista corregida).

\section{Discusión}

Relevancia actual de la lista de Altamirano y Ramírez. A 110 años de la publicación de la lista de árboles y arbustos para repoblar los bosques de la República Mexicana de Altamirano y Ramírez, las causas que los llevaron a desarrollarla están más vigentes que nunca. Altamirano (1897) abordó la necesidad de repoblar los bosques dado el gran deterioro que ya para esa época era considerable dada la intensidad de la explotación de este tipo de ecosistemas. Indiscutiblemente, el daño que se ha causado a los diferentes ecosistemas del país no ha hecho más que agravarse desde finales del siglo XIX (Challenger, 1998). De la misma manera, el interés en las especies nativas para programas de reforestación y de restauración ecológica ha visto un renacimiento después de un período relativamente largo, durante el siglo XX, en el que se privilegió el uso de especies exóticas en nuestro país, en particular varias especies de eucaliptos y casuarinas (Hinke, 2000). En términos históricos, después del esfuerzo pionero de Altamirano y Ramírez podemos destacar el trabajo de Conzatti (1914) de proponer a Quercus acutifolia, Dasycarya grises, Morus alba y M. nigra, entre otras especies para la reforestación del Valle de Oaxaca y las recopilaciones recientes llevadas a cabo por Vazquez-Yanes et al. (1999) y Benítez et al. (2004) que ya se han mencionado.

Una pregunta obligada es en qué medida se han incorporado las especies de la lista de Altamirano y Ramírez a los programas de reforestación en nuestro país. La respuesta es que con excepción de algunas especies de pinos, que han reemplazado sobre todo al uso de especies exóticas, el número de especies nativas que se propagan de manera rutinaria para fines de reforestación es muy limitado. Esto es particularmente notable para las especies del género Quercus, que ocupa el segundo lugar en número de especies (11) en la lista de Altamirano y Ramírez y de que existen experiencias relevantes del uso de especies de encinos en proyectos de restauración ecológica (Bonfil et al., 1997; Bonfil, 1998), aunque también ocurre para otras especies como Trema micrantha (Vázquez-Yanes, 1998), o de especies con capacidad de fijar nitrógeno a través de relaciones simbióticas como los árboles del género Alnus, que han sido ampliamente utilizados para fines de restauración ecológica en diversas partes del mundo (LindigCisneros y Vázquez-Yanes, 1997).

Cuando se lleva a cabo una búsqueda de la literatura destaca que de los géneros considerados por Altamirano y Ramírez, a nivel mundial se han utilizado especies de 40 de estos géneros para proyectos de reforestación, creación de sistemas agrosilvopastoriles o restauración ecológica. Más notable aun es que de las especies de la lista, la mayoría no han sido estudiadas hasta la fecha en términos de sus requerimientos de propagación, su potencial de uso y por lo tanto de su utilidad para repoblar los bosques del país. Es improbable que de las 262 plantas que Altamirano y Ramírez indican propagar por semilla se hayan hecho los estudios necesarios para su utilización en reforestación.

\section{Agradecimientos}

Se agradece la ayuda de los siguientes especialistas para la actualización nomenclatural: Leonardo O. (Apocynaceae), Angélica Cervantes Maldonado (Euphorbiaceae), Mario Sousa Sánchez (Leguminosae), Helga Ochoterena (Rubiaceae) y Fernando Chiang Cabrera (Rutaceae). Los comentarios del Dr. Fernando Chiang mejoraron substancialmente esta contribución.

\section{Literatura citada}

Altamirano, F. 1897. Necesidad de la repoblación de los bosques. Naturaleza II, 2 (app.): 11-22. 
Altamirano, F. y J. Ramírez. 1894. Lista de nombres vulgares y botánicos de árboles y arbustos propicios para repoblar los bosques de la República acompañados de los climas en que vegetan y de la manera de propagarlos. Secretaria de Fomento, México, D.F. 17 págs.

Benítez, G., M. T. P. Pulido-Salas y M. Equihua. 2004. Árboles multiusos nativos de Veracruz para reforestación, restauración y plantaciones. Instituto de Ecología, A.C., SIGOLFO, Conafor. Xalapa, Veracruz, México. 288 pp.

Bonfil, C. 1998. The effects of seed size, cotiledon reserves, and herbivory on seedling survival and growth in Quercus rugosa and Quercus laurina (Fagaceae). American Journal of Botany, 85: 79-87.

Bonfil, C., I. Pisanty, A. Mendoza y J. Soberón. 1997. Investigación y restauración ecológica: El caso del Ajusco Medio. Ciencia y Desarrollo, 135: 14-23.

Bradshaw, A. D. 1982. The creation of nitrogen cicles in derelict land. Philosophical Transaction of the Royal Society of London, 296: 557-561.

Challenger, A. 1998. Utilización y conservación de los ecosistemas terrestres de México; Pasado, presente y futuro. Comisión Nacional para el Conocimiento y Uso de la Biodiversidad, México. D.F.

Conzatti, C. 1914. La repoblación arbórea del Valle de Oaxaca. Secretaría de Fomento, Dirección General de Agricultura, México. Boletín. Estación Agrícola Experimental. Oaxaca 1:1-13.

Correll, D. S. y M. C. Johnston. 1979. Manual of the Vascular Plants of Texas. The University of Texas at Dallas, Texas. $1881 \mathrm{pp}$.

Flora de Veracruz. 1978-2003. Instituto de Ecología, A.C. Xalapa, Veracruz. 129 fascículos.

Flora Neotropica. 1967-1999. Organization for Flora Neotropica, New York Botanical Garden. Bronx, New York. Monograph 1-77.

Flores-Olvera, H. y H. Ochoterena-Booth. 1991. José Ramírez (1852-1904) Vida y Obra. Cuadernos 11. Instituto de Biología, Universidad Nacional Autónoma de México. México, D.F. 102. pp.

Gleason, H. A. y A. Cronquist. 1991. Manual of Vascular Plants of North eastern United States and adjacent Canada. $2^{a}$. ed. New York Botanical Garden, New York. 910 págs.

Hickman, J.C. (ed.). 1993. The Jepson Manual, Higher Plantas of California.University of California Press, Berkeley. 1400 págs.

Hinke, N. 2000. La llegada del eucalipto a México. Ciencias, 58: 60-62.

Bailey, L. 1976. Hortus Third. Macmillan, New York. 1290 pp.

Ibewiro, B., N. Sanginga, et al. 2000. Evaluation of symbiotic dinitrogen inputs of herbaceous legumes into tropical cover-crop systems. Biology and Fertility of Soils, 32: 234-242.

Lindig-Cisneros, R. y C. Vázquez-Yanes. 1997. Los Ailes en la Restauración Ecológica. Ciencia, 48: 31-39.

Martínez, M. 1927. Sinonimia vulgar y científica de las plantas mexicanas. Fondo de Cultura Económica, México. $1220 \mathrm{pp}$.
McVaugh, R. 1983-1993. Flora Novo-Galiciana. University of Michigan Herbarium. 8 vols.

Mcvaugh, R. y J. Rzedowski. 1964-1966. Synopsis of the genus Bursera L. in western México, with notes on the material of Bursera collected by Sessé and Mociño. Kew Bulletin, 18: 317-382.

Pennington, T. D. y J. Sarukhán. 1998. Árboles tropicales de México. Manual para la identificación de las principales especies. Universidad Nacional Autónoma de México y Fondo de Cultura Económica. México, D.F. Segunda edición 521 págs.

Ramírez, J. y G. V. Alcocer. 1902. Sinonimia vulgar y científica de las plantas mexicanas. Secretaría de Fomento, México. 160 págs.

Rzedowski, J. 1986. Vegetación de México. Limusa. Tercera reimpresión, México.

Rzedowski, J. y G. Calderón de Rzedowski (Eds.). 19912003. Flora del Bajío y de Regiones Adyacentes. Instituto de Ecología, A.C. Centro Regional del Bajío, Michoacán. 118 fascículos.

Rzedowski, G. C. De, J. Rzedowski y Colaboradores. 2001. Flora fanerogámica del Valle de México. $2^{\mathrm{a}}$.ed., Instituto de Ecología, A.C. y Comisión Nacional para el Conocimiento y Uso de la Biodiversidad, Pátzcuaro (Michoacán). 1406 pp.

Shreve, F. y I. L. Wiggins. 1964. Vegetation and Flora of the Sonoran Desert.

Stanford University Press, Stanford. 2 vols.

Standley, P. C. 1920-1926. Trees and shrubs of México. Contributions from the

United States National Herbarium. Vol. 23. Parts 1-3 (pp. 1-848), 4-5 (pp. 849-1721).

Standley, P.C. y J. A. Steyermark. 1946. Flora of Guatemala. Fieldiana: Botany Vol. 24, part IV.

Stevens, W. D., C. Ulloa Ulloa, A. Pool y O. M. Montiel (Eds.). 2001. Flora de Nicaragua. Missouri Botanical Garden Press. St. Louis, Missouri. Tomos I-III.

Stopes, C., S. Millington Y L. Woodward. 1996. Dry matter and nitrogen accumulation by three leguminous green manure species and the yield of a following wheat crop in an organic production system. Agriculture Ecosystems and Environment, 57: 189-196.

Vázquez-Yanes, C. 1998. Trema micrantha (L.) Blume (Ulmaceae): A promising neotropical tree for site amelioration of deforested land. Agroforestry Systems, 40: 97-104.

Vázquez-Yanes, C., A. I. Batiz Muñoz, M. I. Alcocer Silva, M. Gual Díaz y C. Sánchez Dirzo. 1999. Árboles y arbustos potencialmente valiosos para la restauración ecológica y reforestación. Reporte técnico del proyecto J084. CONABIO-Instituto de Ecología, UNAM.

Zanoni, T. A. y R. P. Adams. 1979. The genus Juniperus (Cupressaceae) in Mexico and Guatemala: synonymy, key, and distributions of the taxa. Boletín de la Sociedad Botánica de México, 38: 83-121. 


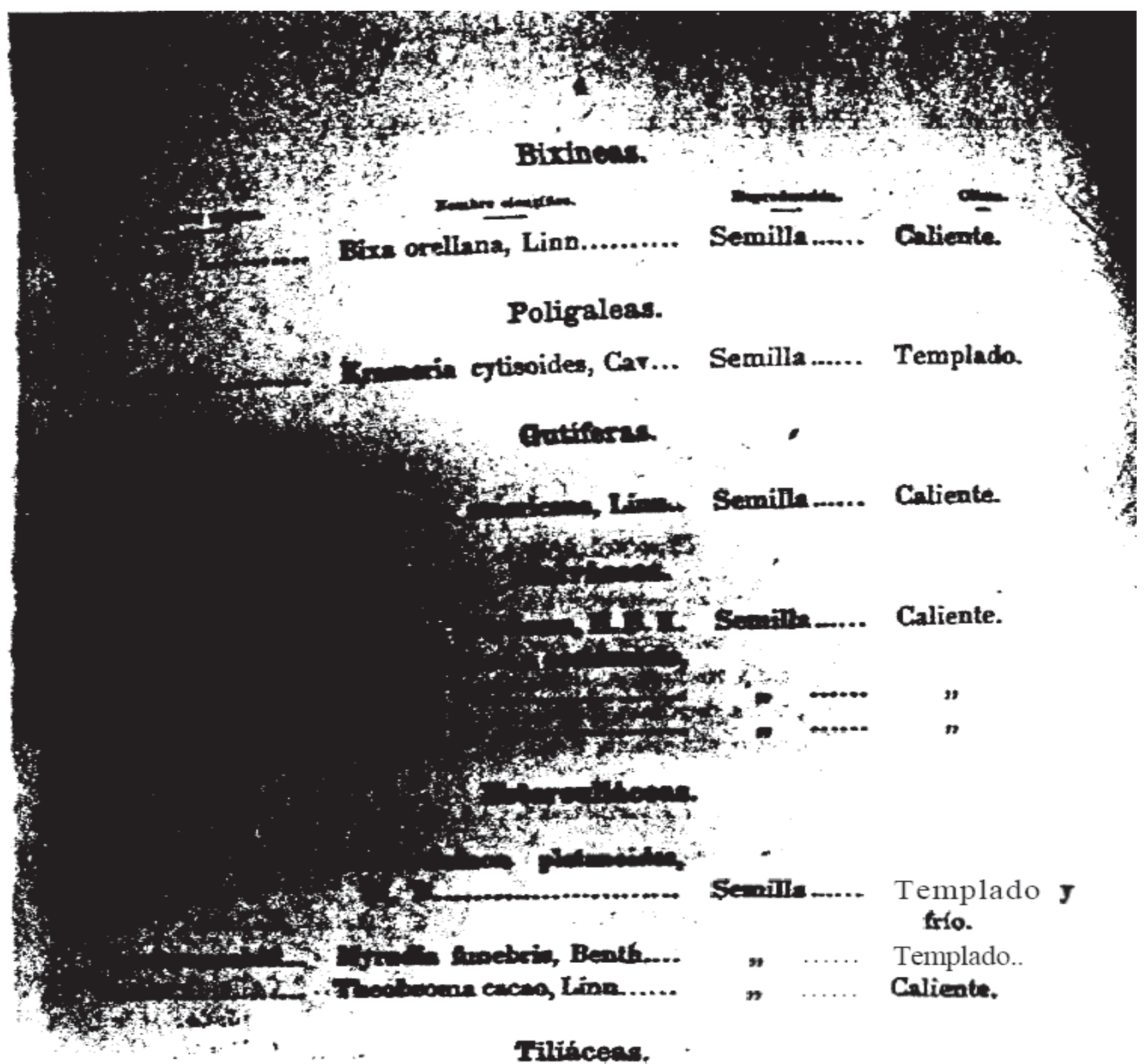

Jonote.

Heliocarpus americanus,

Linn .

Semilla ...... Caliente.

Patastillo

Luher platypetala, A. Rich.

Jolosin............. Heliocarpus arborescens, Sem .

$\eta \ldots . .$.

Templada.

Tilia ó Sirimo...

Tilia mexicana, Benth........

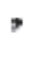

Caliente.

" ....... Templado.

\section{Malpigisceas.}

Nanclie

Bunchosia sp?...

Nanche dulce... .

Byrsonima cotinifolia, $\mathrm{H}$.

Semilla ..... Caliente.

B. K......... 